\title{
Visual Multi-target Tracking
}

\author{
David Ben
}

\begin{abstract}
Speaker following is a difficult assignment and for the most part no scientific arrangement is accessible, particularly for the multi-speaker following framework, which is a high dimensional and non-direct framework. To address this issue, likelihood theory thickness (PHD) filter is utilized by proliferating the PHD rather than the irregular limited set (RFS). As the consecutive Monte Carlo likelihood theory thickness (SMCPHD) filter is anything but difficult to actualize, it has been utilized generally in multi-target following issues. Nonetheless, the presentation of the SMC-PHD filter is influenced by the weight decline issue. In this paper, we will sum up the issue of multi-target following.
\end{abstract}

\section{INTRODUCTION}

The issue of recognition and following of numerous moving speakers in indoor situations utilizing various media (AV) modalities has pulled in an expanding measure of consideration in the most recent decade because of its likely applications in for example programmed camera guiding in video conferencing, singular speaker segregation in multi-speaker conditions, and reconnaissance and observing in security applications. A few difficulties are related with AV following including combination of different modalities, estimation of the variable number of speakers and their states, and managing different conditions, for example, impediment, constrained perspective on cameras, light change and room resonation. This proposition plans to address some portion of these difficulties under the Bayesian structure. This prompts three fundamental commitments summed up as follows.

Multi-speaker following in encased spaces has gotten a lot of enthusiasm for the fields of PC vision and sign preparing, driven by applications, for example, programmed camera controlling in video conferencing [1], singular speaker separating in multi-speaker situations [2], acoustic pillar shaping [3], broad media discourse acknowledgment [4], video ordering and recovery [5], human-PC cooperation [6], and reconnaissance and observing [7] in security applications. Be that as it may, multi-speaker following is a difficult undertaking, all things considered, situations as a few particular issues impact the following procedure, for example, factor number of speakers and their states. The perceptions utilized in multitarget following frequently incorporate arbitrary mistakes, fake comes back from messes, and location misfortune [1]. For instance in visual following, the following outcome is regularly influenced by impediments and the restricted field of perspective on cameras [24], while in sound following, speakers are not generally recognizable when solid foundation clamor and room resonations are introduced in the estimations or when the speakers are quiet.

Early calculation in speaker following regularly utilize single ethical quality, for example, visual information or sound information. The strategy for video-just following [8], [9] is commonly dependable and precise when the objectives are just moving in the camera field of view. Be that as it may, when the objectives are vanished or blocked, the visual following calculation ordinarily lose the objectives [10], [11]. Sound following [12], [13], [14] isn't confined by these impediments, be that as it may, sound information is discontinuous after some time and might be ruined by foundation clamor and room resonations, which may present non-insignificant following mistakes. Aside from that, spatial goals of sound is when all is said in done more terrible than that of video.

To address this issue, the complementarity between various modalities can be abused and a few methodologies have been proposed which can be classified into two strategies as one is deterministic and information driven while the other is stochastic and model-driven [15], [16]. For instance, in view of sound data, speakers can be followed when they are outwardly impeded, while dependent on visual data, speakers can be followed when sound data gets untrustworthy because of the solid foundation clamor. Albeit other multi-modular data, for example, warm vision and laser rangefinders, can likewise be applied to tackle this issue, broad media sensors have been generally utilized because of the ease and the comfort in their establishment [17]. We center around various media information for multi-speaker following.

With the multi-modular data produced by cameras and amplifier clusters, a few calculations have been proposed which can be ordered into two techniques as one is deterministic and information driven while the other is stochastic and model-driven [125], [152]. Deterministic methodologies are frequently considered as an advancement issue by limiting a suitable cost work. The meaning of the cost work is a significant assignment. A typical technique in the writing is mean-move [18], [19] strategy where the cost work is characterized as far as shading similitude estimated by Bhattacharyya separation. The stochastic and model-driven methodologies utilize a state-space approach dependent on the Bayesian structure which is appropriate for preparing multi-modular data [20]. Bayesian induction structure gives a natural route to the estimation of speaker states in a dynamical framework [12]. Be that as it may, tractable answers for the recursive Bayesian estimation issue are just accessible for the straight Gaussian model or the limited state Hidden Markov model (HMM). For nonlinear state estimation,extended Kalman filter (EKF) [13] is frequently utilized. Molecule channel, as an elective strategy, can evaluate the speaker state by a weighted arrangement of arbitrary particles. In any case, it works with the suspicion that the quantity of speakers is known and invariant.

To follow multi-speaker with multi-modalities, information affiliation techniques, for example, joint likelihood information affiliation (JPDA) or numerous speculation following (MHT), 
are expected to run with PF [21] which brings about an expanded computational expense. To follow an obscure and time-variation number of speakers, the irregular limited sets (RFS) strategy initially proposed in [14] has been applied in [15] for multi-speaker following. The RFS strategy gives the establishment to the advancement of an assortment of channels, for example, a Gaussian blend (GM) PHD filter [9], consecutive Monte Carlo (SMC) PHD filter [16], the cardinalized PHD filter [17], and the Generalized named multi-Bernoulli (GLMB) RFS [18]. The SMC-PHD channel, otherwise called the molecule PHD channel, utilizes a lot of irregular particles to assess the back thickness and is applied broadly for multi-target following [16]. This technique, nonetheless, experiences the weight decline issue [19], for example after certain cycles in the molecule spread procedure, the loads of most particles will get immaterial, aside from just a couple of huge loads.

To address the molecule decadence issue, a few variants of the SMC strategies have been proposed with an ideal proposition dissemination, for example, the assistant molecule filter [20], unscented molecule filter [21], helper SMC-PHD filter [22] and unscented assistant cardinalized PHD filter [23]. They misuse the latest estimations or the unscented trans-arrangement to inexact the ideal proposition conveyance and limit the fluctuation of the significance loads. Another notable strategy is the take after move calculation [24], in light of the Markov Chain Monte Carlo technique [25]. The particles delivered by the MCMC cycles speak to sensibly free examples from the objective back. At last, connecting densities [22], [23], [24], [25], [26], [27] have additionally been applied to moderate the weight decadence issue by moving toward the genuine back thickness from the tractable earlier thickness. In spite of its hypothetical style and promising execution, the down to earth usage of this methodology includes confounded estimate of the ideal spanning densities.

As of late, molecule stream has been proposed [29], [30], [31], which moves particles from the earlier appropriation to the back circulation, utilizing a homotopy work which characterizes the progression of manufactured time and embedded for a molecule update at each time period [32]. As indicated by the various suppositions utilized for settling the homotopy work, molecule stream can be isolated into five classes: incompressible molecule stream [33], zero dissemination molecule stream (ZPF) [31], Coulomb's law molecule stream [34], zero-shape molecule stream [35] and non-zero dispersion molecule stream (NPF) [36]. Among them, the ZPFcan be handily executed [37] and it has been generally applied [38], [39], [40]. The ZPF accomplishes better execution for a lower level of estimation clamor [28], [41], where the weight decadence issue is bound to occur, accordingly making ZPF undeniably fit to address this issue.

Molecule stream has been utilized to improve the exactness of the molecule filter [22], and is signified as the molecule stream molecule filter (PFPF). Unique in relation to traditional molecule channels, the PFPF utilizes few particles to accomplish the comparative precision as that for molecule channels with a higher successful example size (ESS) [24]. Be that as it may, for multi-target following, a reliant filter should be applied to each target, which presents the model-information affiliation issue [25]. What's more, earlier information on the quantity of targets is required. In [23], a Gaussian molecule stream

execution of the PHD filter (GPF-PHD) is proposed yielding acceptable exactness in a nonlinear following issue. Be that as it may, in this strategy, the particles are produced for each target, and the computational expense could be high for countless targets and mess. For non-straight and non-Gaussian issues, the helper molecule PHD filter proposed in has preferred execution over the GPF-PHD filter as far as Optimal Subpattern Assignment (OSPA) [25], since it productively appropriates the particles by augmenting the precision of the cardinality estimate

Various media SMC-PHD (AV-SMC-PHD) filter is presented in [8], where sound information are utilized to improve the visual SMC-PHD filter by utilizing the course of appearance (DOA)to re-distribute the enduring and produced particles in the visual SMC-PHD channel. Be that as it may, when speakers don't confront the camera, the back thickness couldn't be refreshed effectively because of the low probability of particles. Another issue is the weight decadence issue, i.e., just few particles have high loads and the powerful example size (ESS)becomes low [42]. Albeit mean-move is utilized to alleviate the weight decline issue by moving particles to the nearby maxima of the estimation work in the scanty AVMSSMC-PHD filter [8], these particles are moved to the pinnacle of the probability thickness and may not precisely speak to the back thickness.

\section{Visual Cues Tracking Modalities}

Visual following of an objective in a picture succession is a troublesome errand, all things considered, situations, as the light conditions may shift and the objective might be blocked by foundation questions or may perform quick and entangled developments [28]. To beat these issues, a few visual highlights, i.e., shading, surface, shape and movement [29] are utilized in existing following frameworks.

An instinctive way to deal with target following is to utilize shading highlights. Hues are regularly utilized in following as they give an extra wellspring of data to recognize targets and object of interests. Various methodologies have been proposed to follow the objective with shading.

A shading blend model is utilized dependent on a Gaussian appropriation to perform following and division in [30]. A following calculation utilizing a versatile blend model is then evolved in [31]. Utilizing shading data, the objective can be handily recognized and followed as long as the delegate shades of the objective is adequately unmistakable from others or the foundation. In that sense, the shading models of the objective should be adapted either already, in a different instatement step [11], or for each new introduced track. Furthermore, the presence of hues might be affected by encompassing or nearby changes in lighting [32].

Another instinctive methodology in following is to utilize form based strategies which track the objective form by utilizing shape coordinating or form development procedures 
[33]. In speaking to the forms, dynamic models can be utilized, for example, snakes, B-splines, and geodesic dynamic shapes, or networks [34]. One general issue in target following is impediment of the objective by objects or different targets. To deal with impediment, just the shape of the chest area can be recognized [35] and followed [36] as opposed to following the form of the entire bodies. This assists with distinguishing a renewed individual since the front and back perspective on the chest areas are unmistakable for various individuals.

One normally utilized signal is surface which is a proportion of the force variety of a surface which measures properties, for example, perfection and consistency [37], [38], [39]. The surface component is concentrated with Gabor wavelet in [40]. The Gabor channels can be utilized as direction and scale tunable edge and line identifiers, and the insights of these small scale includes in a given area are for the most part used to portray the fundamental surface data [41]. As of late, neighborhood examples of picture have gotten expanding enthusiasm for better identification also, acknowledgment. Neighborhood designs that are binarized with a versatile limit, give promising outcomes on different points, for example, face recognition and picture characterization. The Nearby Binary Patterns (LBP) strategy was proposed in [42]. It is an effective surface descriptor characterized as a dark scale invariant surface measure. A favorable position of the LBP is its resistance to enlightenment changes.

Surface is a proportion of the force variety of a surface which measures properties, for example, perfection and normality [37], [38], [39]. Gabor wavelet [40] is presumably the most considered surface element.

The Gabor channels can be considered as direction and scale tunable edge and line indicators, and the insights of these smaller scale includes in a given area are frequently used to describe the basic surface data. As of late, expanding premium is paid on exploring picture's neighborhood designs for better location and acknowledgment.

Particularly, nearby examples that are binarized with a versatile edge give cutting edge results on different subjects, for example, face discovery and picture grouping. In Ojala et al. [42], built up a productive surface descriptor, called Local Binary Patterns (LBP). The LBP surface examination administrator is characterized as a grayscale invariant surface measure, got from a general meaning of surface in a nearby neighbor-hood. The most significant property of the LBP administrator is its resilience against brightening changes. Another similarly significant trademark is its computational straightforwardness.

Another cue used in tracking, especially in indoor environments, is motion which is a very strong cue of human presence. This cue can be extracted by using foreground detection. A simple method of foreground detection is to compute the difference frame by frame. Although it has been used in multi-modal tracking systems [43], it fails when the person is stationary since it does not provide any foreground information.

Visual Object Tracking Based on Local Steering Kernels and Color Histograms

[28]
Appearance based following techniques utilize the visual data of the item projection on the picture plane, i.e., shading, surface, and shape, just as data on the 2-D object movement [8]. These strategies manage straightforward article changes, for example, relative ones, including interpretation and revolution. Notwithstanding, they are touchy to light changes.

Form based following strategies track the article form by utilizing shape coordinating orcontour-advancement procedures [9]. Forms can be spoken to by dynamic models, for example, snakes, B-splines, and geodesic dynamic shapes, or networks [10], empowering the following of both unbending and nonrigid items. So as to manage in part impeded articles, following calculations join impediment location and estimation strategies.

Highlight based strategies perform object following by following a lot of highlight focuses, which speak to the article [11]. These followed highlights are then gathered, as indicated by their relationship in the past casing. These techniques perform well in incomplete impediment, just as in following extremely little items. The serious issue of featurebased strategies is the right differentiation between the objective article and foundation highlights. At last, half and half techniques for object following endeavor the benefits of the previously mentioned strategies, by joining at least two following strategies [12]. As a rule, include based techniques are utilized first, for object recognition and confinement. At that point, area based methods are utilized to follow its parts. The primary disservice of these strategies is their high computational intricacy.

Multimodal Probabilistic Person Tracking and Identification in Smart Spaces

Hues are broadly utilized for following assignments when all is said in done as they offer an extra wellspring of data to separate between objects of intrigue. They offer the preferred position that the item or individual of intrigue can be handily identified and followed as long as its delegate hues or "surface" is adequately unmistakable from others and the foundation. The drawback to utilizing surface or shading models is that they should be learned in either already, in a different instatement step [62], or on-the-fly for each new introduced track. Further, the presence of hues may shift extensively starting with one camera sensor then onto the next, or even through an ideal opportunity for one same sensor, as it is unequivocally impacted by surrounding or neighborhood changes in lighting.

The Scale Invariant Feature Transform (SIFT) [44] is likewise broadly utilized as an element in following applications. It changes picture information into scale-invariant directions comparative with neighborhood highlights. The SIFT extricates unmistakable invariant highlights from pictures and manages solid coordinating between various perspectives on an item. The SIFT highlight is invariant to interpretation, turn, scaling, mess, lighting and impediment. Likewise, it is hearty for coordinating over a generous scope of relative twists, change in $3 D$ perspective, expansion of commotion, and change in light. The highlights are for the most part unmistakable which permit a solitary element to be effectively coordinated with high likelihood against an enormous database of highlights from a picture database that incorporates nu- 
merous pictures. In any case, the SIFT coordinating rate and acknowledgment execution can be significantly diminished in loud situations with non-unbending targets [45].

\section{CONCLUSION}

Among the obvious signs clarified above, shading signals have been utilized all through this proposal because of their simple usage and low multifaceted nature. Shading data is utilized by taking the histogram of potential focuses at the introduction step as reference pictures and afterward utilizing them in location and following of the objective. In the writing, the RGB or HSV shading histogram model is normally utilized [46]. In our examination, HSV is picked since it is seen to be increasingly powerful to enlightenment variety.

\section{REFERENCES}

[1] Q. Liu, Y. Rui, A. Gupta, and J. J. Cadiz, "Automating camera management for lecture room environments," in Proc. the SIGCHI Con. Human Factors in Computing Systems. ACM, 2001, pp. 442-449.

[2] F. Talantzis, A. Pnevmatikakis, and A. G. Constantinides, "Audiovisual active speaker tracking in cluttered indoors environments," IEEE Trans. Systems, Man, and Cybernetics, Part B: Cybernetics, vol. 38, pp. 799807, Jun. 2008.

[3] M. Wölfel and J. W. McDonough, "Combining multi-source far distance speech recognition strategies: Beamforming, blind channel and confusion network combination," in Proc. INTERSPEECH, Sep. 2005, pp. 3149-3152.

[4] G. Potamianos, C. Neti, and S. Deligne, "Joint audio-visual speech processing for recognition and enhancement," in Proc. Int. Conf. AudioVisual Speech Processing, 2003.

[5] M. R. Naphade, T. Kristjansson, B. Frey, and T. S. Huang, "Probabilistic multimedia objects (multijects): A novel approach to video indexing and retrieval in multimedia systems," in Proc. IEEE Int. Con. Image Processing, 1998, pp. 536-540.

[6] S. T. Shivappa, B. D. Rao, and M. M. Trivedi, "Audio-visual fusion and tracking with multilevel iterative decoding: Framework and experimental evaluation," IEEE Journal of Selected Topics in Signal Processing, vol. 4, pp. 882-894, Oct. 2010.

[7] A. Hampapur, L. Brown, J. Connell, A. Ekin, N. Haas, M. Lu, H. Merkl, and S. Pankanti, "Smart video surveillance: exploring the concept of multiscale spatiotemporal tracking," IEEE Signal Processing Magazine, vol. 22, no. 2, pp. 38-51, Mar. 2005.

[8] D. A. Ross, J. Lim, R.-S. Lin, and M.-H. Yang, "Incremental learning for robust visual tracking," Int. Journal of Computer Vision, vol. 77, no. 1-3, pp. 125-141, 2008.

[9] M. M. Trivedi, H. S. Kohsia, and I. Mikic, "Dynamic context capture and distributed video arrays for intelligent spaces," IEEE Trans. Systems, Man and Cybernetics, Part A: Systems and Humans, vol. 35, no. 1, pp. 145-163, Jan. 2005.

[10] M. Isard and J. MacCormick, "Bramble: A Bayesian multiple-blob tracker," in Proc. Int. Con. Computer Vision, vol. 2, 2001, pp. 34-41.

[11] O. Lanz, "Approximate bayesian multibody tracking," IEEE Trans. Pattern Analysis and Machine Intelligence, vol. 28, no. 9, pp. 14361449, Sep. 2006.

[12] M. F. Fallon and S. J. Godsill, "Acoustic source localization and tracking of a time-varying number of speakers," IEEE Trans. Audio, Speech, and Language Processing, vol. 20, no. 4, pp. 1409-1415, 2012.

[13] I. Potamitis, H. Chen, and G. Tremoulis, "Tracking of multiple moving speakers with multiple microphone arrays," IEEE Trans. Speech and Audio Processing, vol. 12, no. 5, pp. 520-529, Sep. 2004.

[14] N. Roman and D. Wang, "Binaural tracking of multiple moving sources," IEEE Trans. Audio, Speech, and Language Processing, vol. 16, no. 4, pp. 728-739, May 2008.

[15] J. Sullivan and J. Rittscher, "Guiding random particles by deterministic search," in Proc. Int. Con. Computer Vision, vol. 1. IEEE, 2001, pp. 323-330.

[16] S. K. Zhou, R. Chellappa, and B. Moghaddam, "Visual tracking and recognition using appearance-adaptive models in particle filters," IEEE Trans. Image Processing, vol. 13, no. 11, pp. 1491-1506, 2004.
[17] J. Vermaak, M. Gangnet, A. Blake, and P. Perez, "Sequential Monte Carlo fusion of sound and vision for speaker tracking," in Proc. IEEE Int. Conf. Computer Vision (ICCV), vol. 1, 2001, pp. 741-746.

[18] D. Comaniciu and P. Meer, "Mean shift: A robust approach toward feature space analysis," IEEE Trans. Pattern Analysis and Machine Intelligence, vol. 24, no. 5, pp. 603-619, May 2002.

[19] D. Comaniciu, V. Ramesh, and P. Meer, "Kernel-based object tracking," IEEE Trans. Pattern Analysis and Machine Intelligence, vol. 25, no. 5, pp. 564-577, May 2003.

[20] D. Gatica-Perez, G. Lathoud, I. McCowan, J.-M. Odobez, and D. Moore, "Audio-visual speaker tracking with importance particle filters," in Proc. IEEE Int. Con. Image Processing, vol. 3, Sep. 2003, pp. 25-28.

[21] D. Schulz, W. Burgard, D. Fox, and A. B. Cremers, "Tracking multiple moving targets with a mobile robot using particle filters and statistical data association," in Proc. Int. Conf. Robotics and Automation, vol. 2. IEEE, 2001, pp. 1665-1670.

[22] Y. Liu, W. Wang, J. Chambers, V. Kilic, and A. Hilton, "Particle flow smc-phd filter for audio-visual multi-speaker tracking," in International Conference on Latent Variable Analysis and Signal Separation. Springer, 2017, pp. 344-353.

[23] Y. Liu, W. Wang, and Y. Zhao, "Particle flow for sequential monte carlo implementation of probability hypothesis density," in 2017 IEEE International Conference on Acoustics, Speech and Signal Processing (ICASSP). IEEE, 2017, pp. 4371-4375.

[24] Y. Liu, A. Hilton, J. Chambers, Y. Zhao, and W. Wang, "Non-zero diffusion particle flow SMC-PHD filter for audio-visual multi-speaker tracking," Proc. IEEE Int. Conf. Acoustics, Speech and Signal Processing (ICASSP), 2018.

[25] Y. Liu, W. Wang, and V. Kilic, "Intensity particle flow smc-phd filter for audio speaker tracking," arXiv preprint arXiv:1812.01570, 2018.

[26] Y. Liu, V. Kılıç, J. Guan, and W. Wang, "Audio-visual particle flow smc-phd filtering for multi-speaker tracking," IEEE Transactions on Multimedia, vol. 22, no. 4, pp. 934-948, 2019.

[27] Y. Liu, Q. Hu, Y. Zou, and W. Wang, "Labelled non-zero particle flow for smc-phd filtering," in ICASSP 2019-2019 IEEE International Conference on Acoustics, Speech and Signal Processing (ICASSP). IEEE, 2019, pp. 5197-5201.

[28] O. Zoidi, A. Tefas, and I. Pitas, "Visual object tracking based on local steering kernels and color histograms," IEEE Trans. Circuits and Systems for Video Technology, vol. 23, no. 5, pp. 870-882, May 2013.

[29] C. Yang, R. Duraiswami, and L. Davis, "Efficient mean-shift tracking via a new similarity measure," in Proc. IEEE Computer Society Con. Computer Vision and Pattern Recognition, vol. 1, 2005, pp. 176-183.

[30] Y. Raja, S. J. McKenna, and S. Gong, "Segmentation and tracking using colour mixture models," in Proc. Asian Conferenece on Computer Vision. Springer, 1998, pp. 607-614.

[31] S. J. McKenna, Y. Raja, and S. Gong, "Tracking colour objects using adaptive mixture models," Image and Vision Computing, vol. 17, no. 3, pp. 225-231, 1999.

[32] K. Bernardin, "Multimodal Probabilistic Person Tracking and Identification in Smart Spaces," Ph.D. dissertation, Karlsruhe University, 2009.

[33] A. Yilmaz, X. Li, and M. Shah, "Contour-based object tracking with occlusion handling in video acquired using mobile cameras," IEEE Trans. Pattern Analysis and Machine Intelligence, vol. 26, no. 11, pp. 1531-1536, 2004.

[34] Y. Wang and O. Lee, "Active mesh-a feature seeking and tracking image sequence representation scheme," IEEE Trans. Image Processing, vol. 3, no. 5, pp. 610-624, Sep. 1994.

[35] A. S. Micilotta, E.-J. Ong, and R. Bowden, "Real-time upper body detection and 3D pose estimation in monoscopic images," in Proc. European Con. Computer Vision. Springer, 2006, pp. 139-150.

[36] K. Bernardin, T. Gehrig, and R. Stiefelhagen, "Multi-level particle filter fusion of features and cues for audio-visual person tracking," in Multimodal Technologies for Perception of Humans. Springer, 2008, pp. 70-81.

[37] R. Fergus, P. Perona, and A. Zisserman, "Object class recognition by unsupervised scale-invariant learning," in Proc. IEEE Computer Society Con. Computer Vision and Pattern Recognition, vol. 2, 2003, pp. 264271.

[38] J. Shotton, J. Winn, C. Rother, and A. Criminisi, "Textonboost for image understanding: Multi-class object recognition and segmentation by jointly modeling texture, layout, and context," Int. Journal of Computer Vision, vol. 81, no. 1, pp. 2-23, 2009.

[39] J. Winn, A. Criminisi, and T. Minka, "Object categorization by learned universal visual dictionary," in Proc. Int. Con. Computer Vision, vol. 2 , 2005, pp. 1800-1807. 
[40] B. S. Manjunath and W.-Y. Ma, "Texture features for browsing and retrieval of image data," IEEE Trans. Pattern Analysis and Machine Intelligence, vol. 18, no. 8, pp. 837-842, 1996.

[41] H. Yang, L. Shao, F. Zheng, L. Wang, and Z. Song, "Recent advances and trends in visual tracking: A review," Neurocomputing, vol. 74, no. 18 , pp. 3823-3831, 2011

[42] T. Ojala, M. Pietikäinen, and T. Mäenpää, "Multiresolution gray-scale and rotation invariant texture classification with local binary patterns," IEEE Trans. Pattern Analysis and Machine Intelligence, vol. 24, no. 7, pp. 971-987, 2002.

[43] P. Perez, J. Vermaak, and A. Blake, "Data fusion for visual tracking with particles," Proc. the IEEE, vol. 92, no. 3, pp. 495-513, 2004.

[44] D. G. Lowe, "Distinctive image features from scale-invariant keypoints," Int. Journal of Computer Vision, vol. 60, no. 2, pp. 91-110, 2004.

[45] Y. Ke and R. Sukthankar, "PCA-SIFT: A more distinctive representation for local image descriptors," in Proc. IEEE Computer Society Con. Computer Vision and Pattern Recognition, vol. 2, 2004, pp. 506-513.

[46] L. Sigal, S. Sclaroff, and V. Athitsos, "Estimation and prediction of evolving color distributions for skin segmentation under varying illumination," in Proc. IEEE Con. Computer Vision and Pattern Recognition, vol. 2, 2000, pp. 152-159. 